\title{
Is a simple rational classification of maxillary sinus dimensions applicable?
}

Kristina Bert|1 ${ }^{1,2}$, René-Bernard Mick², Patrick Heimel ${ }^{3,4,5}$, André Gahleitner $^{6}$, Andreas Stavropoulos ${ }^{1}$, Christian Ulm² $^{2}$

1 Dept. of Periodontology, Faculty of Odontology, University of Malmö, Sweden

Division of Oral Surgery, School of Dentistry, Medical University of Vienna, Austria

Karl Donath Laboratory for Hard Tissue and Biomaterial Research, Medical University of Vienna, Austria

Austrian Cluster for Tssue Regeneration, Vienna, Austria

6 Dept. of Diagnostic Radiology, Division of Osteoradiology, General Hospital, Medical University of Vienna, Austria

\section{Background}

Maxillary sinus floor augmentation (MSFA) with autologous bone and/or bone substitutes is a reliable procedure to develop an implant site. Loss of augmentation volume during the healing period seems to be dependent on the type of graft material and possibly on the sinus dimensions/morphology; e. a larger distance between the buccal and to a longer distance angiogenic/osteogenic factors and cells need to cover to populate the graft.

\section{Aim}

1. Assess the bucco-palatal width of the maxillary sinus in the premolar \& molar region.

2. Assess any possible differences due to tooth region, gender, tooth-gap extent \& residual alveolar bone dimensions.

3. Attempt a rational, simple sinus classification based on its bucco-palatal width.

\section{Methods and Materials}

- Inclusion criteria: 1) maxillary CT scan; 2) sites presenting $>5 \mathrm{~mm}$ height of the maxillary sinus on the $\mathrm{CT} ; 3$ ) no previous augmentation procedures; and 4) no oro-antral communication.

- 2 groups: 1) edentulous \& 2) partially dentate (i.e., 1- or 2-tooth gap).

- A central orthoradial multiplanar reconstruction slice from each edentulous tooth site was evaluated on the following parameters: alveolar bone height $(A B H)$, alveolar bone area (ABA), alveolar bone width (ABW) $2 \mathrm{~mm}$ apical to the alveolar crest (ABW2), ABW at the sinus floor (ABWS), bucco-palatal sinus width (SW) and sinus area (SA) at a level 2, 4, 6, 8 and $10 \mathrm{~mm}$ above the sinus floor.
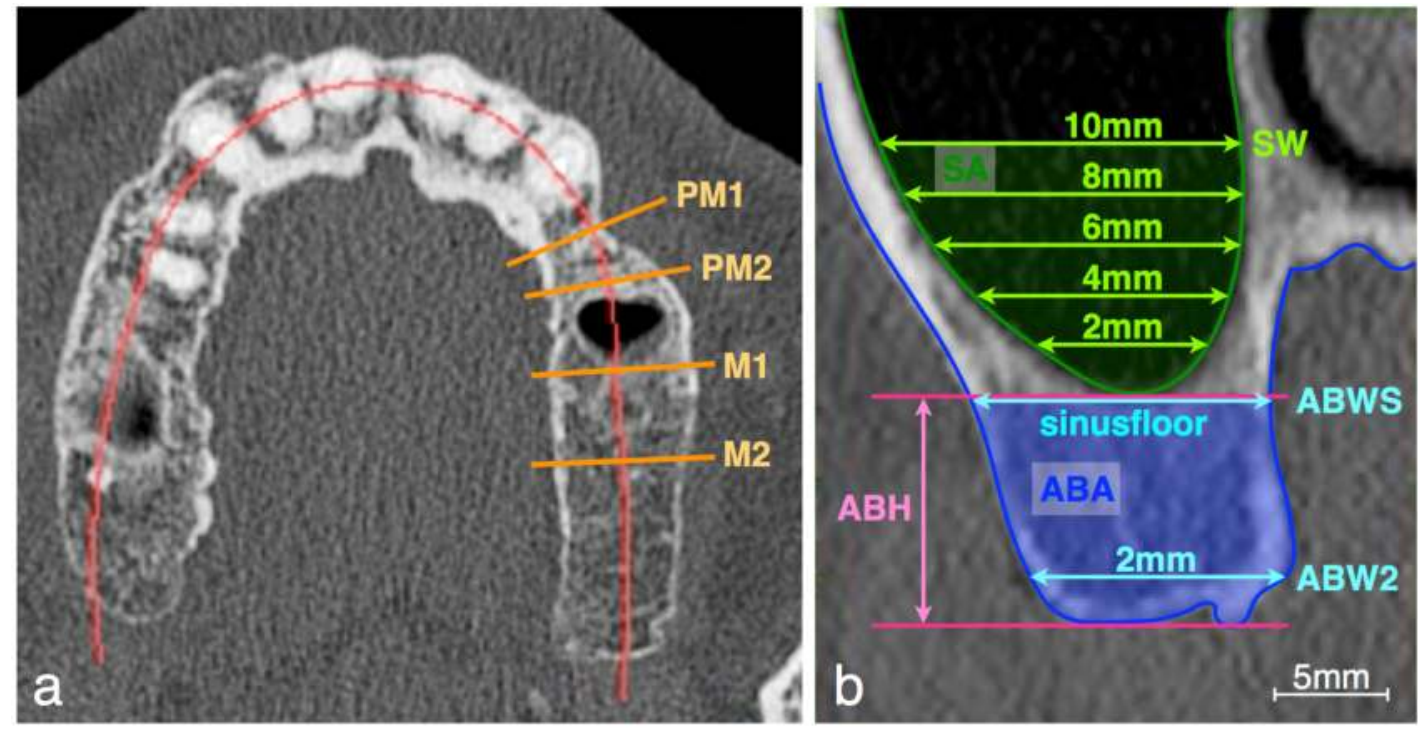

- The sinus was classified as narrow, average, and wide based on mean values (i.e., $<25^{\text {th }}, 25-75^{\text {th }},>75^{\text {th }}$ percentile and $<33^{\text {rd }}, 33-67^{\text {th }}$ $>67^{\text {th }}$ percentile)

- The frequency distribution of a SW $<10,10$ to 15 , or $>15 \mathrm{~mm}$ at the different tooth sites and height levels was assessed.

\section{Results}

- 86 partially dentate and 76 edentulous quadrants contributed with 383 tooth sites (58 first and 97 second premolars; 129 first and 99 second molars)

- Alveolar bone and sinus parameters did not differ depending on toothgap extent and gender, but differed significantly depending on tooth site; i.e., $A B H$ and $A B A$ was smallest at the first molar, and ABW, ABWS, SW, and SA were significantly larger at the molars.

- ABW correlated significantly with SW and SA.

- A high variability in SW among the various levels within the same tooth site, as well as among the different tooth sites within the same person was observed; i.e., in $>50 \%$ of the cases, the sinus classification (i.e., narrow, average, or wide) varied depending on SW height level and among tooth sites within each patient.

\begin{tabular}{|c|c|c|c|c|c|}
\hline \multirow{2}{*}{\multicolumn{2}{|c|}{ Sinus classification }} & PM1 & PM2 & M1 & M2 \\
\hline & & \multicolumn{4}{|c|}{$\frac{1}{\%}$} \\
\hline \multicolumn{2}{|c|}{ Unclassified } & 6.8 & 7.2 & 9.3 & 5.1 \\
\hline \multicolumn{2}{|c|}{ Narrow $-<25^{\text {th }}$} & 20.3 & 20.6 & 19.4 & 23.5 \\
\hline \multicolumn{2}{|c|}{ Average $-25-75^{\text {th }}$} & 47.5 & 51.5 & 48.8 & 48.0 \\
\hline \multicolumn{2}{|c|}{ Wide $->75^{\text {th }}$} & 25.4 & 20.6 & 22.5 & 23.5 \\
\hline \multirow{3}{*}{$\begin{array}{c}\text { No. of different } \\
\text { classifications/site }\end{array}$} & 1 & 49.2 & 39.2 & 34.1 & 42.9 \\
\hline & 2 & 49.2 & 54.6 & 59.7 & 52.0 \\
\hline & 3 & 1.7 & 6.2 & 6.2 & 5.1 \\
\hline \multicolumn{2}{|c|}{ Unclassified } & 10.2 & 13.4 & $\overline{11.6}$ & 9.2 \\
\hline \multicolumn{2}{|c|}{ Narrow $-<33^{\text {rd }}$} & 27.1 & 28.9 & 27.9 & 34.7 \\
\hline \multicolumn{2}{|c|}{ Average $-33-67^{\text {th }}$} & 27.1 & 26.8 & 29.5 & 28.6 \\
\hline \multicolumn{2}{|c|}{ Wide $->67^{\text {th }}$} & 35.6 & 30.9 & 31.0 & 27.6 \\
\hline \multirow{3}{*}{$\begin{array}{c}\text { No. of different } \\
\text { classifications/site }\end{array}$} & 1 & 40.7 & 34.0 & 32.6 & 41.8 \\
\hline & 2 & 52.5 & 52.6 & 55.0 & 48.0 \\
\hline & 3 & 6.8 & 13.4 & 12.4 & 10.2 \\
\hline
\end{tabular}

Similarly, the frequency distribution of $\mathrm{SW}<10,10-15$, or $>15 \mathrm{~mm}$ varied significantly among tooth sites.

\begin{tabular}{|c|c|c|c|c|c|c|}
\hline \multirow{2}{*}{ Height } & \multirow{2}{*}{ Sinus width } & PM1 & PM2 & M1 & M2 & \multirow{2}{*}{$\begin{array}{l}\text { p-value } \\
\left(x^{2} \text {-test }\right)\end{array}$} \\
\hline & & \multicolumn{4}{|c|}{$\%$} & \\
\hline \multirow{3}{*}{$2 \mathrm{~mm}$} & $<10 \mathrm{~mm}$ & 100 & 97.9 & 94.6 & 93.9 & \multirow{3}{*}{0.277} \\
\hline & $10-15 \mathrm{~mm}$ & 0 & 2.1 & 5.4 & 5.1 & \\
\hline & $>15 \mathrm{~mm}$ & 0 & 0 & 0 & 1.0 & \\
\hline \multirow{3}{*}{$6 \mathrm{~mm}$} & $<10 \mathrm{~mm}$ & 77.6 & 43.3 & 8.5 & 15.2 & \multirow{3}{*}{$<0.001$} \\
\hline & $10-15 \mathrm{~mm}$ & 22.4 & 52.6 & 69.8 & 74.7 & \\
\hline & $>15 \mathrm{~mm}$ & 0 & 4.1 & 21.7 & 10.1 & \\
\hline \multirow{3}{*}{$10 \mathrm{~mm}$} & $<10 \mathrm{~mm}$ & 67.6 & 32.9 & 0 & 7.1 & \multirow{3}{*}{$<0.001$} \\
\hline & $10-15 \mathrm{~mm}$ & 29.7 & 45.9 & 34.9 & 36.4 & \\
\hline & $>15 \mathrm{~mm}$ & 2.7 & 21.1 & 65.1 & 56.5 & \\
\hline
\end{tabular}

4 patient cases with different sinus morphologies based on the $<25^{\text {th }}$ $25-75^{\text {th }},>75^{\text {th }}$ percentile of the SW at each specific height level and tooth site classifying the sinus as narrow (class 1; pink), average (class 2; green), and wide (class 3 ; light blue). In general, these 4 cases can be described as (a-d) overall narrow sinus, (e-h) wide sinus at the premolars, but narrow at the molars, $(\mathrm{i}-\mathrm{I})$ overall wide sinus, and $(m-p)$ narrow sinus at the premolars, but wide at the molars.
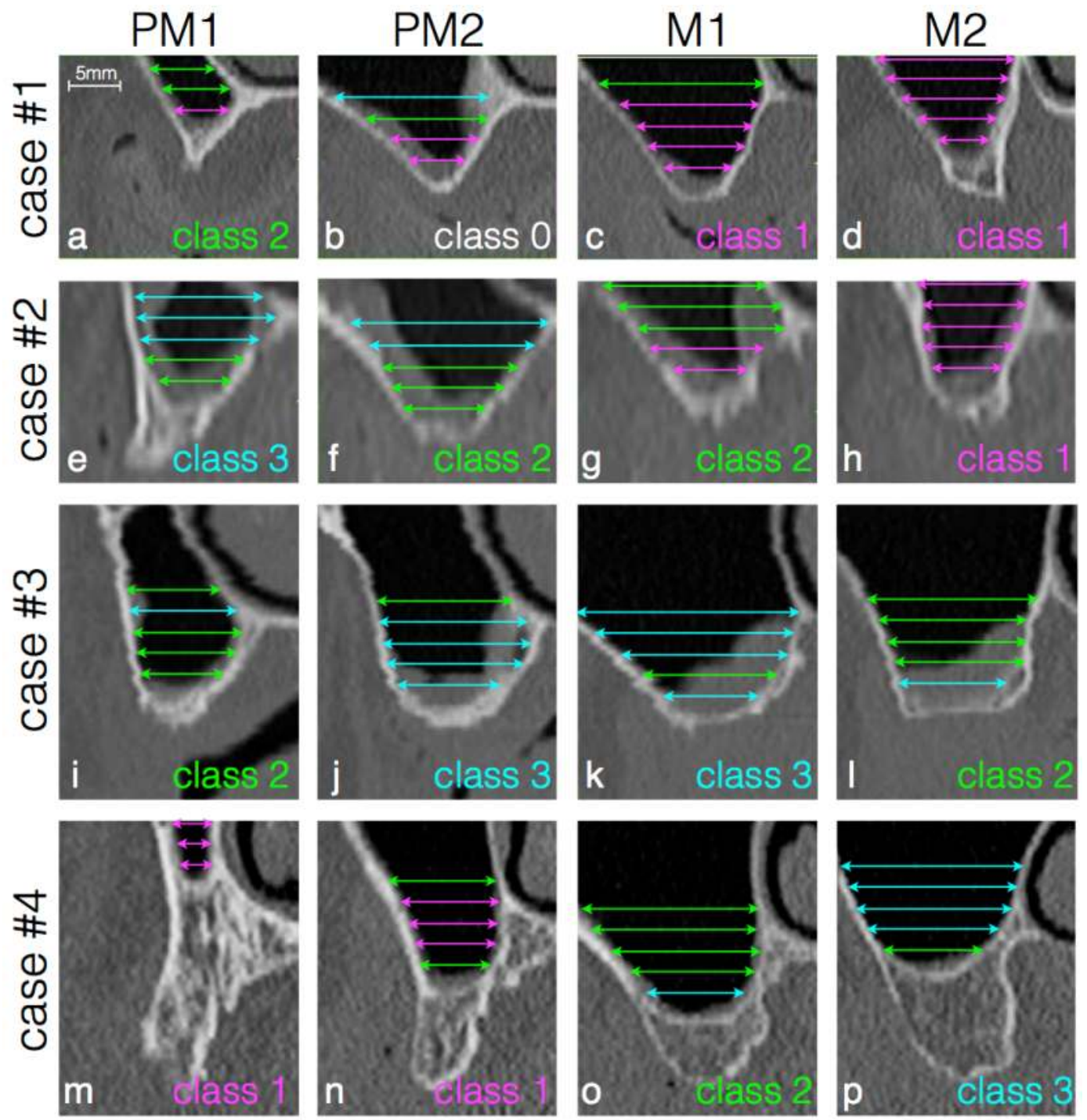

\section{Conclusions}

Maxillary bucco-palatal sinus width varies significantly among the various height levels within the same tooth site and among the different tooth sites within the same person. A rational, simple classification of each sinus as narrow, average, or wide was not applicable. 